\title{
GESTIÓN DEL MEDIO AMBIENTE URBANO. RESIDUOS QUE SE GENERAN EN LA ACTIVIDAD DE LA CONSTRUCCIÓN. DIRECTIVA U.E "DEMOLITION WASTE"
}

\section{( URBAN ENVIRONMENT MANAGEMENT. WASTES PRODUCED BY CONSTRUCTION WORKS "DEMOLITION WASTE". E.C. DIRECTIVE)}

Luis Fontanet Sallán, Dr. Ingeniero Industrial. EUR. ING.

Justo García Navarro, Dr. Arquitecto. Prof. Titular de la UPM

Fecha de recepción: 26-III-96 ESPAÑA

\section{RESUMEN}

El número de agentes contaminantes que inciden y degradan el Medio Ambiente se multiplica día tras dia. Una gran mayoría de ellos tienen un origen común: son residuos generados por el hombre en su actividad cotidiana.

El sector de la construcción, uno de los principales motores de la economía, interpreta también un papel protagonista a la hora de generar residuos. Sin embargo, en España y hasta la fecha, la preocupación por su tratamiento, reutilización o, cuando menos, correcto vertido, ha sido prácticamente inexistente.

La posible Directiva comunitaria que, a este respecto, podria ver la luz el presente año 1996, aborda en profundidad la problemática generada por los residuos producidos en la actividad de la construcción y plantea la necesidad de su adecuado tratamiento.

Este artículo esboza lo que debiera ser el camino a seguir.

\section{Introducción}

El Medio Ambiente Urbano está siendo gravemente afectado por las actividades que en el mismo se desarrollan. La atmósfera, medio principal de nuestro hábitat, es afectada por laemisión de diversos contaminantes; las consecuencias de la alta densidad de tráfico de vehículos han ocasionado

\section{SUMMARY}

The number of polluting agents that falls and degrades the environment, is increasing from day to day. A lot of them have a common origin: they are residues generated by the man during his daily activity.

The construction sector, one of the most important engines of the economy, also plays a main role at time to generate residues. However, in Spain, until nowadays, the worry for its treatment, new use or, at least, right spilt, has been nearly inexistent.

The possible E.C. Directive that could be published this year, treats the problematic that has been generated by the waste produced by the construction activity and plans the necesity of its right treatment.

This paper shows the right way that could be followed. la adopción, en muchas ciudades, de medidas especiales. La contaminación acústica se convierte en un impacto desagradable que pretende convivir con los ciudadanos. El depósito, transporte y tratamiento final de los residuos que se generan, es causa de una buena parte de las serias preocupaciones de las autoridades responsables y del público en general. No quedan libres de este tipo de impactos las 
fuentes de abastecimiento de agua, los espacios recreacionales intraurbanos y periurbanos $y$, en las zonas costeras, las playas, en las que la contaminación se produce por el vertido de aguas residuales urbanas e industriales.

En particular, la actividad de la CONSTRUCCIÓN es potencial y realmente causante, en algunos casos, de la contaminación atmosférica y acústica del Medio Ambiente Urbano, así como generadora de gran cantidad de residuos. Todas las acciones que se desarrollan durante el proceso de ejecución de una obra y que se integrarían en lo que actualmente se conoce como "ciclo de vida" -es decir, desde la explotación de canteras, el movimiento de tierras y el transporte de materiales, por ejemplo, hasta el acabado final de las obras-, producen impactos sobre el Medio Ambiente, de tal forma que laUnión Europea ha considerado a los residuos generados en esta actividad como pertenecientes al grupo denominado Flujo de Residuos Prioritarios, objeto actualmente de la elaboración de la Directiva "DEMOLITION WASTE". La aplicación de la misma cambiará, sin lugar a dudas, muchos de los aspectos no considerados o no tratados adecuadamente hasta ahora.

Por otro lado, la Federación de la Industria Europea de la Construcción (FIEC) ya eligió el Medio Ambiente como tema básico de su Asamblea anual de 1992 y, en el seno de su Comisión Técnica, creó una Subcomisión específica de análisis de la problemática Medioambiental.

\section{Los residuos en la actividad de la construcción}

En razón a las diferentes modalidades que se consideren, así comoal emplazamientoy situación general de las obras, la actividad de la construcción genera en cualquier caso residuos cuya tipología y cuantificación tiene unas características especiales. Una gran proporción de los mismos puede clasificarse como "residuos inertes" y, por tanto, pueden ser gestionados como tales.

En nuestro país, los residuos de la construcción (Obra Nueva y Demolición ) -RCD- no se separan en origen, salvo casos excepcionales de algún material cuyo valor justifique dicha separación, o bien constituya un beneficio adicional para el encargado de la obra. Estos residuos, en forma de "todo uno" son transportados hasta el lugar de destino y depositados, no siempre, en vertederos más o menos controlados. Constituyen, por otra parte, buenos materiales para el relleno de espacios y regularización de superficies, si se emplean de forma ordenada y programada. Ejemplo de este tipo de actuaciones son algunas de las obras de infraestructura de las instalaciones para los Juegos Olímpicos de Barcelona. No obstante, los modelos de gestión que se aplican en nuestro país distan mucho de la práctica habitual en otros países europeos, a pesar de laaparición en el mercado de medios más adecuados para realizar las diferentes operaciones de acopio, selección y transporte: contenedores, vehículos especiales con sistemas "autocargantes", etc.

Los datos de producción, composición, etc., necesarios inicialmente para comenzar a estudiar y determinar en consecuencia futuras políticas de actuación y optimización, a pesar de figurar, en parte, cuantificados en los cuadros adjuntos, son los únicos existentes y deben por tanto estimarse con cierta reservas, ya que estos residuos no han sido objeto de control hasta ahora. Deberán transcurrir al menos cinco años para poderlos contrastar y actualizar debidamente.

Los valores que figuran en los mencionados cuadrosresumen se han obtenido del Documento Informativo preparado por ARGUS e ITU (consultoras encargadas de la coordinación técnica de las reuniones de los grupos de trabajo) para la COMMISSION OF THE EUROPEAN COMMUNITIES, Grupo de trabajo "Construction and Demolition Waste", encargado de la elaboración de la propuesta de Directiva.

Resulta interesante la reflexión en torno a determinados valores de los citados cuadros. Sin lugar a dudas, los datos más fiables y que representan una mayor aproximación a la realidad de situación actual son los que corresponden a Alemania; los datos de Holanda pueden ser también bastante fiables y muy próximos a la realidad.

CUADRO N ${ }^{\circ} 1$

\begin{tabular}{||l|c|c|l||}
\hline \multicolumn{4}{|c|}{ Producción de Residuos RCD } \\
\hline Pais & $\begin{array}{c}\text { Producción } \\
\text { (miles) }\end{array}$ & $\begin{array}{c}\text { Producción } \\
\text { per cápita } \\
\text { (kg/hab.año) }\end{array}$ & Observaciones \\
\hline Alemania & 53.000 & 880 & $\begin{array}{l}\text { Sólo antigua } \\
\text { RFA } \\
\text { Bélgica }\end{array}$ \\
Dinamarca & 7.000 & 700 & (1) \\
España & 6.500 & 1.275 & (2) \\
Francia & 11.000 & 285 & Datos de \\
Grecia & 30.000 & 580 & 1978 \\
\hline Holanda & N.D. & N.D. & \\
Irlanda & 14.000 & 940 & (1) \\
Italia & 400 & 110 & Datos de \\
Luxemburgo & 1.750 & 50 & 1977 (3) \\
Portugal & 48 & 185 & Datos de \\
Reino Unido & 50.000 & 900 & 1976 (3) \\
\hline \hline
\end{tabular}

N.D. Dato no disponible

(1): No incluye tierras de excavación ni RCD residuos de construcción y demolición- provenientes de obras públicas

(2): Sólo incluye residuos de demolición de edificios

(3): Incluye residuos de demolición y de construcción de nuevos edificios 
CUADRO $\mathrm{N}^{\circ} 2$

\begin{tabular}{|c|c|c|c|c|}
\hline \multicolumn{5}{|c|}{$\begin{array}{l}\text { Composición de los RCD en diversos países (1990) } \\
\text { (\% en peso) }\end{array}$} \\
\hline Fracción & $\begin{array}{l}\text { Alemania } \\
\text { (1) }\end{array}$ & $\begin{array}{l}\text { Dinamarca } \\
\text { (2) }\end{array}$ & Holanda & $\begin{array}{l}\text { Reino } \\
\text { Unido }\end{array}$ \\
\hline Hormigón & 34,00 & 40,00 & 44,00 & 50,00 \\
\hline Fábricas & 32,00 & 52,00 & 27,00 & 40,00 \\
\hline $\begin{array}{l}\text { Materia } \\
\text { Granular }\end{array}$ & & & & 20,00 \\
\hline $\begin{array}{l}\text { Fracciones } \\
\text { mezcladas }\end{array}$ & & & & 3,40 \\
\hline Tejas & & & & 0,60 \\
\hline Madera & 13,00 & 8,00 & 2,30 & 1,00 \\
\hline Metales & 1,40 & 0,30 & & \\
\hline $\begin{array}{l}\text { Prod.bitu- } \\
\text { minosos }\end{array}$ & & & & 0,20 \\
\hline Plásticos & 12,00 & & & 0,30 \\
\hline $\begin{array}{l}\text { Fracción } \\
\text { residual }\end{array}$ & 9,00 & 0,80 & 8,70 & \\
\hline
\end{tabular}

(1): Composición correspondiente a RCD de obras de edificación

(2): Composición correspondiente a residuos de demolición de edificios

(3): Composición correspondiente a residuos de demolición
CUADRO N $\mathrm{N}^{\circ} 3$

\begin{tabular}{|c|c|c|}
\hline \multicolumn{3}{|c|}{ Plantas de tratamiento de RCD en la Unión Europea (1990) } \\
\hline País & $\mathbf{N}^{0}$ de plantas & Observaciones \\
\hline Alemania & más de 300 & Incluye la antigua RDA \\
\hline Bélgica & 40 & $\begin{array}{l}\text { Información de Flandes. El 75\% } \\
\text { de las plantas son fijas }\end{array}$ \\
\hline Dinamarca & 17 & 7 fijas, 7 móviles y 3 semimóviles \\
\hline España & 1 & Localizada en Barcelona \\
\hline Francia & 10 & \\
\hline \multicolumn{3}{|c|}{ Estimación de las existencias } \\
\hline País & $N^{0}$ de plantas & Observaciones \\
\hline Grecia & N.D. & \\
\hline Holanda & 60 & 45 fijas y 15 móviles \\
\hline Irlanda & 0 & \\
\hline Italia & 5 & La mayor parte son móviles \\
\hline Luxemburgo & N.D. & \\
\hline Portugal & 0 & \\
\hline Reino Unido & 9 & 3 fijas, y al menos, 6 móviles \\
\hline
\end{tabular}

N.D.: Dato no disponible

\section{CUADRO $\mathrm{N}^{0} 4$}

\begin{tabular}{||c|c|c|c|c|c|c||}
\hline \multicolumn{5}{||c||}{ Evolución de tarifas de vertido de RCD (Ptas./T) } \\
\hline DINAMARCA & \multicolumn{3}{c|}{ HOLANDA } \\
\hline AÑo & 1987 & 1991 & 1993 & 1988 & 1990 & 1993 \\
\hline Tarifa & 800 & 3.500 & 3.900 & 1.600 & 3.200 & 6.500 \\
\hline
\end{tabular}

momento apunta hacia el empleo de estos productos como material de relleno en la remodelación de espacios con una morfología adecuaday, en cualquier caso, a su vertido, bajo control permanente, en lugares adecuados. Con ello se evitaría el vertido indiscriminado e incontrolado $\mathrm{y}$, en especial, los vertidos de mezclas de residuos inertes con toda clase de residuos, lo que viene siendo práctica habitual en una gran mayoría de los casos.

Normalmente, en España el árido fabricado con material de origen cuesta menos que el árido que se obtendría por tratamiento y acondicionamiento de los residuos de demolición. Este hecho resulta decisivo a la hora de dar viabilidad práctica y real a una política de reciclaje, por mucho que la Administración adopte una política activa de proteccionismo que impulse estas acciones.

La política comunitaria tiende, en general, a llevar a cabo cuantas acciones sean necesarias para eliminar prácticamente el vertedero como solución a aplicar en la fase final de la gestión de estos residuos. Las figuras número 1 (Evolución prevista en el tratamiento de los residuos) y 2 (Estrategia aconsejable) son una representación gráfica de las líneas de trabajo apuntadas en la Directiva comunitaria. 


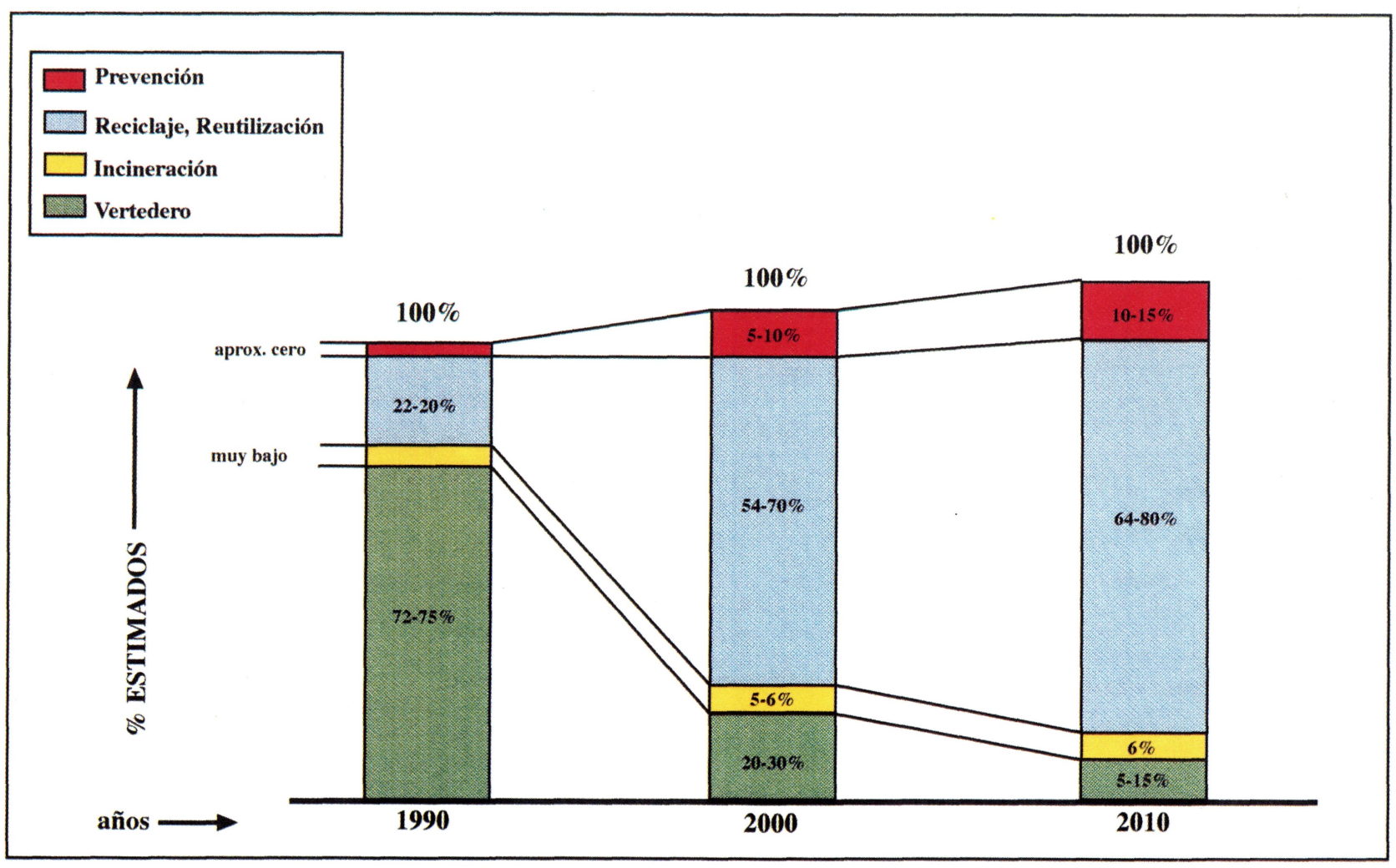

Fig. 1.- Evolución prevista en el tratamiento de los residuos.

Todo ello responde, por una parte, a la lógica reacción producida por las auténticas y graves deficiencias que en la gestión de vertederos se han producido en todos los países; por otra, a la presión que determinados países con tecnología propia y específica para el tratamiento y reciclaje ejercen, dada la imperiosa necesidad de "vender" a otros países sus instalaciones. No hay que olvidar que el vertedero controlado constituye una solución más, con la que hay que contar en determinadas circunstancias y que, en casi todos los casos, complementa a otras soluciones de tratamiento-reciclaje en las que se genera una fracción cuya eliminación no admite, por el momento, la adopción de soluciones asequibles.

\section{Planteamientos y objetivos}

Los planteamientos y objetivos de una posible Directiva comunitaria deberán abordar primordialmente una serie de aspectos fundamentales. No se pretende realizar una relación exhaustiva, pero sí reflejar el espíritu que debería transmitirse en su desarrollo.

En todas las políticas de Gestión de Residuos se da lógicamente mucha importancia a lo que se considera como preventivo, es decir, a las medidas y disposiciones a adoptar antes de que los materiales y productos se conviertan en residuos, lo que lógicamente comporta una labor de examen y análisis previo de los materiales a emplear en una obra, desde el punto de vista de su procedencia, durabilidad y composición.

En esta línea, algunas de estas medidas deberán ser las siguientes:

A) El número y diversidad de materiales actualmente empleados en la construcción, debe reducirse

La heterogeneidad de los componentes de un residuo dificulta extraordinariamente las labores de selección y posterior tratamiento. En la construcción se emplean con frecuencia materiales que son componentes de elementos estructurales, acabados, cerramientos, etc., que cumplen con la misma misión, incluso estética, pero en los que cada fabricante emplea materias primas y procesos que hacen diferente de los demás al producto final.

En la actualidad resulta prácticamente imposible llegar a catalogar, analizar y clasificar los diferentes productos que 
ESTRATEGIA ACONSEJABLE

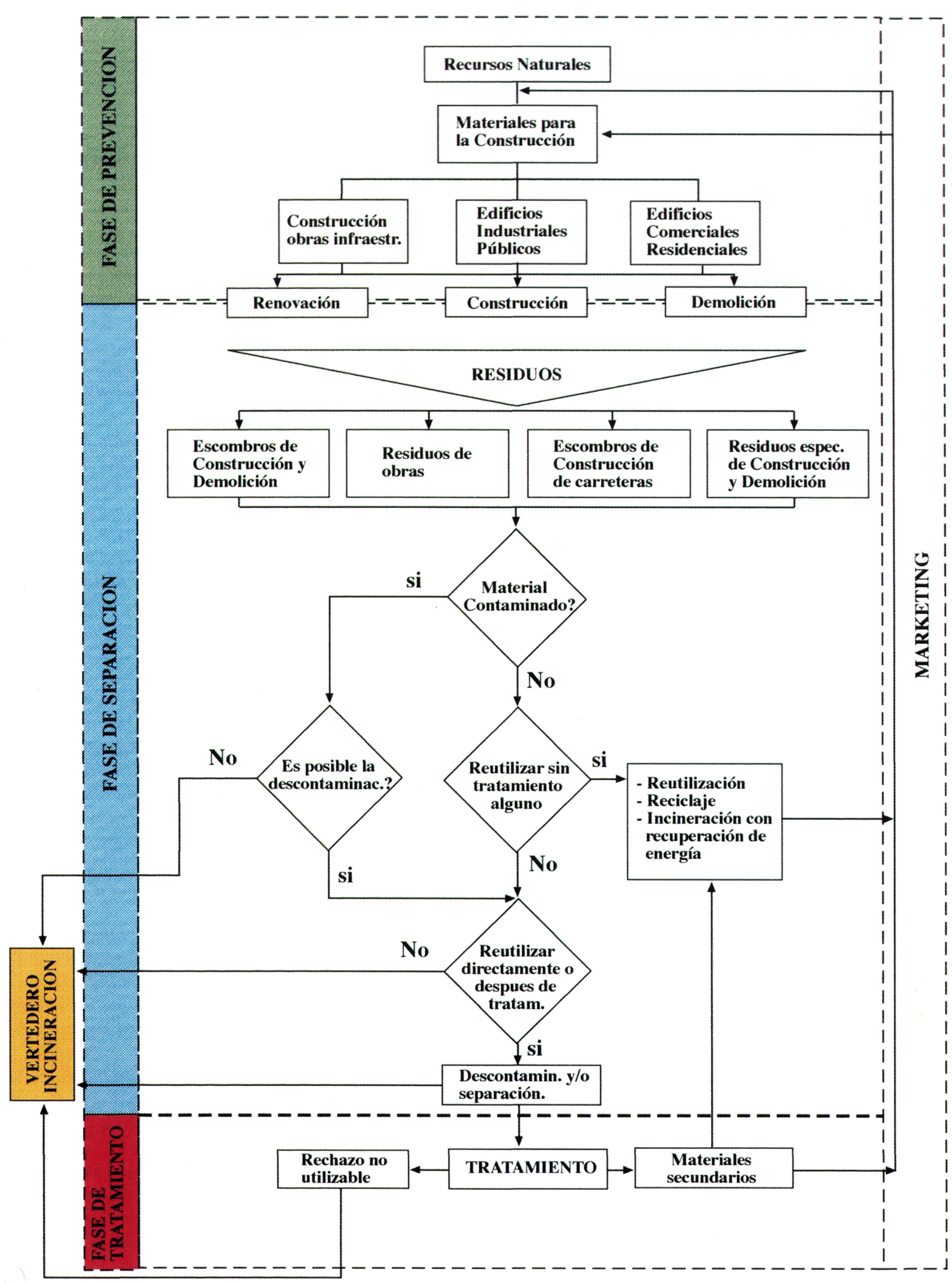

Fig. 2.- Estrategia aconsejable. 
tienen su aplicación en el sector de la edificación. Sin ocasionar grandes traumas puede llegarse, en sucesivas acciones coordinadas, a disminuir del orden de un $25 \%$ la diversidad de determinados productos que, al final de su vida útil, cuando sean objeto de derribo, se convertirán en residuos

B) En la elección de los materiales que se propongan en obra deberá considerarse los costes e impactos ambientales posibles de su "ciclo de vida"

El "ciclo de vida" comprende lo que podríamos denominar, más gráficamente, como el período que va de la cuna a la tumba, es decir, desde que el material se fabrica, hasta que es llevado a su último destino, que bien podría ser el de su reutilización. En todo este ciclo se producen una serie de operaciones cuya influencia y correspondiente repercusión económica se han tenido en cuenta, hasta ahora, de forma parcial

De ahora en adelante, la Directiva pretende establecer que en este tipo de operaciones hay que considerar y valorar en lo posible, por ejemplo, los costes negativos del transporte del material a su destino final, el coste de su tratamiento, así como los costes de los posibles impactos producidos a la atmósfera y causados por los sucesivos transportes.

Naturalmente, en el caso de que el material tenga una posible aplicación como reciclado, podría llegar a considerarse en el coste total un valor residual cuantificable y recuperable

C) En el diseño de las nuevas realizaciones se tendrá en cuenta, además de lo antedicho, la disposición adecuada para que, en el momento de la demolición, ésta pueda llevarse a cabo en la forma más racional y que permita, en lo posible, que la separación de los diferentes materiales se haga al menor coste y con la máxima eficacia

Es evidente que en este caso la adopción, siempre que ello sea posible, de una estructura prefabricada y de fácil montaje debe facilitar, a su vez, el "desmontaje" al llegar el momento de derribo.

Actualmente, determinados montajes de elementos y componentes de una edificación se ejecutan de forma que la inclusión o unión de un material con otro diferente resulte lo más "compacta" posible, dificultando las labores de separación de ambos cuandollega el momento. Es decir, que los edificios se están construyendo sin pensar que algún día puede que haya que desmontarlos para un mejor aprovechamiento de lo existente.

Por otra parte, de los detalles de montaje y ejecución de los diferentes materiales y unidades de obra no se conserva la documentación que permita llevar a cabo, llegado el momento, una demolición controlada.
D) Las demoliciones serán realizadas por empresas responsables, que deberán estar autorizadas

Aunque ahora no se incluya en el texto de una futura Directiva U.E., parece evidente que debe ser así. No obstante, todavía en algunos países las demoliciones de edificios son realizadas por empresas que, aun siendo especializadas, tienen escasa preparación, no cuentan con técnicos responsables y llevan a cabo estos trabajos improvisando las decisiones en cada caso.

En el Grupo de Trabajo se ha planteado asimismo la necesidad de que cada demolición debe ser objeto de un proyecto específico, redactado por técnico competente, en el que además de las lógicas medidas de Seguridad, se contemplará la tipología de los diferentes materiales, su posible selección y destino, ya sea de tratamiento para su reutilización o para depósito final en vertedero autorizado

E) Actualmente los materiales procedentes de derribo tienen una consideración y un trato despectivo. Habráque divulgar el uso y aplicación de estos materiales, destacandosusventajasmedioambientalesy, desdeluego, propiciar su empleo bajo condiciones técnicas y de seguridad, según la normativa en vigory a un precio asequible. En situaciones de autentica desventaja para los productos susceptibles de reciclado-reutilización, se ha llegado a proponer que sean gravados los precios de materias vírgenes con tasas especiales

Cada país presenta al respecto una problemática diferente En España, por ejemplo, se dispone en buena parte del territorio de espacios aptos para albergar residuos que sean inertes, aprovechando-esta circunstancia para remodelar la orografía del área elegida. En un país como Holanda, es lógico que esta circunstancia no se presente $y$, por tanto, el confinamiento de residuos tenga un elevado coste; por otra parte, la inexistencia de canteras para la extracción de áridos obliga al reciclado de determinados materiales

Es evidente que acciones de divulgación y de protección lógica y coherente que propicien el reciclaje de determinados materiales son de utilidad para la política de actuación de la U.E. en materia Medioambiental, que tiene como objetivo prioritariola conservación de los recursos naturales.

F) En los Pliegos de Condiciones, especialmente en obras de la Administración, se deberá recomendar el empleo de materiales procedentes de reciclaje

Éste precisamente puede ser uno de los puntos clave para el desarrollo de esta política y para la puesta en marcha de determinados sectores industriales que desarrollen instalaciones para el reciclado de materiales procedentes de demolición. Hay ya en nuestro país precedentes interesantes con las pruebas y ensayos que se han realizado en la Comunidad Catalana para el empleo de materia- 
les procedentes del triturado de neumáticos fuera de uso, en mezclas asfálticas para carreteras.

Es evidente que la aplicación de la Directiva, y su correspondiente trasposición a nuestra legislación, va a repercutir en muchos de los aspectos que se contemplan actualmente en los proyectos, así como en la realización de obras y demoliciones.

Comunidades Autónomas como Cataluña y el País Vasco ya han legislado al respecto (la Generalitat de Catalunya, en el Diario Oficial $n^{\circ} 1931$, del lunes 8 de agosto de 1994, publicó el "Decreto regulador de los escombros y otros residuos de la Construcción", en el que se recogen muchos de los aspectos aquí enumerados), habiéndose incluso constituido empresas especializadas (por ejemplo, y también en Cataluña, es el caso de Gestora de Runes de la Construcció, S.A.).

En esta misma línea, y con motivo de la celebración del Feria CONSTRUMAT del año 1993, en Barcelona, ya se celebraron unas jornadas técnicas cuyo tema principal era "La problemática de los residuos en la industria de la construcción". Temas como la panorámica de los residuos de la construcción y su procesamiento para el reciclado, y la restitución de morfologías con aprovechamiento de materiales de derribo, inertes, fueron debatidos ampliamente.

G) El mecanismo adecuado para el establecimiento de una normativa armonizada para la gestión de los residuos de construcción y demolición será la aprobación de una Directiva de obligatoria aplicación en los diferentes estados miembros de la U.E.

Sus dos principales objetivos deberán ser la prevención y la valorización de los residuos. Para llegar a conseguir los niveles adecuados será necesario actuar en las siguientes áreas:

- Educación y Formación.

- Legislación y Reglamentación.

- Gestión de los residuos en los propios lugares de construcción y demolición.

- Tratamiento, Transporte y Eliminación de los residuos.

- Planificación de la Gestión de los residuos.

En esta tarea deberán estar implicadas tanto las Administraciones como numerosos organismos, empresas y técnicos:

- Diseñadores, Constructores y Empresas del ramo.

- Empresas especializadas en la valorización, transporte y eliminación de residuos.

- Administraciones Centrales y Autonómicas y Corporaciones Locales.

- Fabricantes y Proveedores de los materiales empleados en construcción.

- Estados miembros de la U.E.

- Asociaciones Profesionales europeas.

- Organizaciones de Consumidores y de Protección del Medio Ambiente.

- La propia Comisión Europea.

H) Los Estados miembros de la U.E. deberán adoptar una nomenclatura y clasificación común que sirva como base para lagestión de los residuos de construccióny demolición, y para la recogida y notificación de datos relativos a la producción de residuos

El último borrador del Grupo de Trabajo incluye ya la siguiente clasificación:

- Código CED 170100: Hormigón, tejas, ladrillos y material cerámico.

- Código CED 170201: Madera.

- Código CED 170203: Materiales plásticos.

- Código CED 170300: Productos asfálticos y betunes

- Código CED 170400: Metales y aleaciones.

- Código CED 170500: Tierras y lodos de drenajes.

- Código CED 170600: Materiales empleados en el aislamiento.

- Código CED 170700: Residuos de construcción y demolición, mezclados.

I) La Unión Europea deberá fomentar subvencionando las acciones que se desarrollen sobre Gestión y Tratamiento de residuos.

Es decir, Proyectos para la recogida, estudio y difusión de datos relativos a la valorización, así como Proyectos de Investigación que traten preferentemente de:

1. Determinación de los obstáculos existentes a la práctica generalizada de la valorización.

2. Adaptación de los procesos y metodologías existentes para su posible adopción por industrias de la construcción de la U.E.

3. Puesta a punto de nuevas técnicas de valorización y gestión general de estos residuos.

4. Localización de nuevos empleos en la construcción para las materias recuperadas.

J) Se deberán crear tasas especiales para aplicar a los vertederos, de las que una parte importante deberá ir destinada a la promoción y valorización de los residuos de construcción y demolición

K) Deberá planificarse la gestión de los residuos, considerando y analizando el "ciclo de vida" de los diferentes productos $y$ materiales 
L) Se deberá recomendar, a los fabricantes de materiales de construcción y proveedores de materiales, el establecimiento de fichas técnicas medioambientales, que incluyan la siguiente información:

- Tipo de producto o material.

- Descripción del producto.

- Lista de componentes.

- Porcentaje de materiales valorizados.

- Categoría (código CED) para su eliminación.

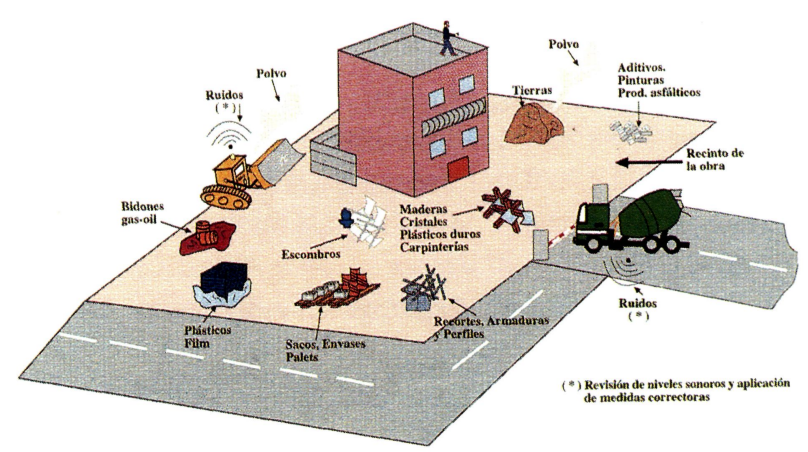

Fig. 3.- Gestión de los residuos generados en las actividades de construcción y demolición.
- Opciones de valorización y eliminación.

\section{El futuro}

Para políticas y actuaciones de este tipo, el futuro empieza hoy. Las medidas que se deban tomar para atajar los efectos contaminantes de agentes tan agresivos como los residuos de construcción y demolición deberán contemplar y ampliar lo aquí apuntado. Sirva como apuesta por planteamientos esperanzadores la actuación publicada en el Warmer Bulletin, en su número de noviembre de 1995, referente a un edificio situado en Odense (Dinamarca), en la que se explica cómo su demolición proporcionó materiales que se reutilizaron en una nueva edificación que se construyó en un solar contiguo

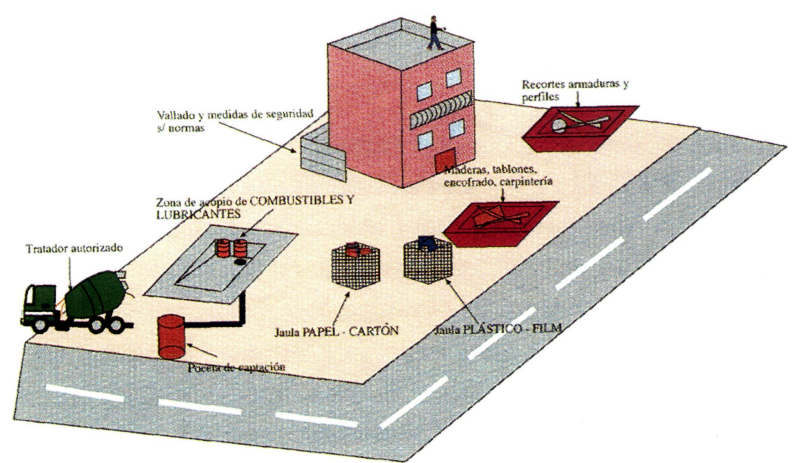

Fig. 4.- Separación y acopio in situ. Acondicionamiento de espacios. 